\title{
Composition Floristique, Diversité Et Structure Des Espèces Forestières Alimentaires De La Région De Sikasso Au Sud Du Mali
}

\author{
Amadou Male Kouyate, \\ Ismaila Diarra, \\ Programme Ressources Forestières, \\ Institut d'Economie Rurale (IER), Sikasso, Mali
}

Rabiou Habou,

Faculté des Sciences de Diffa, Niger

Doi:10.19044/esj.2020.v16n12p156 URL:http://dx.doi.org/10.19044/esj.2020.v16n12p156

\section{Résumé}

Le présent travail a été initié dans le but d'évaluer la composition floristique, la diversité spécifique et la structure des espèces forestières locales alimentaires en vue de lutter contre la malnutrition. Il a été réalisé dans des villages de quatre massifs forestiers naturels appartenant à deux zones agroécologiques au Sud du Mali, soit deux villages par zone. Au niveau de chaque massif forestier, les données ont été collectées dans 10 placettes de $50 \mathrm{~m}$ x 50 $\mathrm{m}$ suivant un échantillonnage systématique à un degré. Dans chaque placette, tous les individus ligneux ont été mesurés. Les données dendrométriques ont été collectées sur chaque arbre (diamètre à hauteur de poitrine appelée en anglais « diameter at breast height » en abrégé « d.b.h. » et la hauteur totale des tiges). Au niveau de chaque placette, le comptage de l'effectif total des jeunes plants (diamètre à hauteur de poitrine $<3 \mathrm{~cm}$ ) a été effectué dans cinq placeaux de $25 \mathrm{~m}^{2}$ suivant un échantillonnage systématique à deux degrés. Les relevés effectués ont permis de recenser 89 espèces ligneuses appartenant à 73 genres et 30 familles botaniques. Les espèces alimentaires représentent 34,83 $\%$ des espèces inventoriées. Des différences significatives de densité, de hauteur totale et de surface terrière moyenne ont été observées entre les massifs forestiers. La densité la plus élevée est observée au niveau du massif forestier de Badogo (71 arbres/ha) et la plus faible au niveau de Sorobasso (24 arbres/ha) qui présente le plus fort taux de régénération $(2,88 \%)$. L'analyse des structures en classe de diamètre des populations d'espèces alimentaires les plus abondantes (Saba senegalensis, Vitellaria paradoxa, Lannea acida, Lannea microcarpa, Lophira lanceolata, Parkia biglobosa et Borassus aethiopum) a montré des tendances variables. Des stratégies de domestication 
et de multiplication végétative des espèces s'avèrent nécessaires pour assurer la disponibilité de la ressource en vue de lutter contre la malnutrition.

Mots clés : Alimentation, Disponibilité, Espèces Forestières, Inventaire, Sud Du Mali

\title{
Floristic Composition, Diversity and Structure of Edible Forest Species in Southern Mali
}

\author{
Amadou Male Kouyate, \\ Ismaila Diarra, \\ Programme Ressources Forestières, \\ Institut d'Economie Rurale (IER), Sikasso, Mali
}

Rabiou Habou,

Faculté des Sciences de Diffa, Niger

\begin{abstract}
The present work was launched to assess the floristic composition and structure of the native forest food species in order to tackle malnutrition. It was carried out in four natural forest from villages in two agro-ecological zones (two villages per agro-ecological zone) in southern Mali. In each natural forest, data were collected in 10 plots of 50 x 50 m using sampling systematic to one degree. In each plot, all tree were measured. Dendrometric data were collected on each tree (diameter at breast height or d.b.h and total stem height). In each plot, the total number of seedlings (diameter at breast height $<3 \mathrm{~cm}$ ) was carried out within five $25 \mathrm{~m}^{2}$ plots using sampling systematic to two degrees. The inventories were recorded 89 native forest species belonging to 73 genera and 30 botanical families. Native edible species were represented $34.83 \%$ of the overall inventoried species. Significant differences were observed between natural forest for average density per hectare, average height and average basal area. The high density was observed in Badogo (71 trees.ha $\left.{ }^{-1}\right)$, while the less in Sorobasso (24 trees.ha ${ }^{-1}$ ) which present the high regeneration rate $(2.88 \%)$. The analysis of population diameter structures for Saba senegalensis, Vitellaria paradoxa, Lannea acida, Lannea microcarpa, Lophira lanceolata, Parkia biglobosa and Borassus aethiopum showed variable trends. Domestication and vegetative propagation strategies of the species are needed to ensure the availability of the resource to combat malnutrition.
\end{abstract}


Keywords: Availability, Forest species, Forest inventory, Mali

\section{Introduction}

La région de Sikasso est située au Sud du Mali. Sa superficie forestière est estimée à 7153600 ha (DNEF, 2014). Cette partie est considérée comme l'une des zones boisées du Mali avec 26 forêts classées, 2 aires protégées, 2 zones d'intérêt cynégétique et 23 forêts communautaires (DREF, 2013). Le domaine forestier protégé occupe environ les $2 / 3$ des formations forestières semi-naturelles de la région de Sikasso. Il est caractérisé par des savanes arbustives, arborées et boisées, des forêts claires et des galeries forestières (ARS, 2011). Les récentes études ont révélé que cette région renferme 277 espèces forestières dont 250 espèces locales et 27 exotiques qui appartiennent à 182 genres et 58 familles. Les familles les plus représentées sont les Fabaceae, les Combretaceae et les Rubiaceae. Les genres les plus représentés sont Acacia et Ficus (Fagui, 2015). Les enquêtes menées en 2014 dans la région ont montré que 30 espèces forestières locales sont en voie d'extinction des terroirs villageois (Fagui, 2015). Parmi ces espèces, note Balanites aegyptiaca (L.) Delile, Borassus aethiopum Mart., Cordyla pinnata (Lepr. ex A.Rich.) Milne-Redh., Elaeis guineensis Jacq., Pentadesma butyracea Sabine, Raphia sudanica A. Chev. Pourtant, ces espèces jouent un rôle alimentaire inestimable pour les populations locales.

La surface des formations semi-naturelles est passée de $55177 \mathrm{~km}^{2}$ à $38183 \mathrm{~km}^{2}$ entre 1987 et 2014 , soit une perte de $32 \%$. Contrairement aux formations semi-naturelles, la surface à dominante agricole est passée de $15229 \mathrm{~km}^{2}$ à $33353 \mathrm{~km}^{2}$ pour la même période (DNEF, 2014). Cette partie du Mali dispose de 6000000 ha de terres qui sont favorables à l'agriculture et l'élevage. On y trouve 14079 ha de bas-fonds et plaines aménagés sur un potentiel de 150000 ha aménageables (CPS/SDR, 2015). Ces écosystèmes abritent un potentiel élevé en plantes alimentaires qui peut jouer un rôle important dans l'amélioration de la sécurité alimentaire. Les enquêtes conduites dans la région de Sikasso sur les plantes alimentaires ont révélé plus de 87 plantes sauvages alimentaires. Ces espèces sont largement utilisées par les populations pour des besoins alimentaire et thérapeutique. Les fruits sont les organes les plus consommés suivis des feuilles et des fleurs (Diarra et al., 2016). Ces formes d'exploitation, opérées quotidiennement par les populations, présentent des conséquences sur le renouvellement des peuplements. En effet, le ramassage des fruits appauvrit la banque séminale édaphique et compromet la régénération des espèces.

Aujourd' hui, cette partie du Mali est considérée comme une importante zone d'élevage avec un cheptel estimé à 1971006 bovins, 1110895 ovins, 1158989 caprins (DRPIA, 2019). En dépit de cet important potentiel, la 
région de Sikasso présente le plus fort taux de malnutrition au Mali, soit $30 \%$ (INSTAT, 2016), d'où le paradoxe de Sikasso (Dury et Bocoum, 2012). Cette malnutrition, due surtout à une alimentation inadéquate, se manifeste par le retard de croissance, la morbidité et l'anémie chez les femmes en âge de procréation.

Pour lutter contre cette malnutrition, il urge de valoriser ces plantes forestières alimentaires et d'accroître la disponibilité d'aliments hautement nutritifs à faible coût. Toutefois, la capacité des aliments forestiers à contribuer à la sécurité alimentaire et à une meilleure nutrition comme éléments de régimes alimentaires durables reste peu exploitée (Vinceti et al., 2013). C'est pour cette raison que le présent travail a été initié pour évaluer la disponibilité des espèces forestières locales alimentaires, car une bonne compréhension du milieu constitue la base de toute activité forestière bien comprise (Sorg, 2000).

\section{Matériel et méthodes}

Milieu d'étude

Le Mali abrite quatorze régions naturelles, six zones agro-climatiques et quarante-neuf zones agro-écologiques selon la classification du PIRT (1986). La région de Sikasso appartient à trois zones agro-climatiques (soudanien nord, soudanien sud et guinéen nord) selon la mêe PIRT (1986), alors que la classification de White (1982) indique deux zones agroécologiques (soudanienne et guinéenne). Les sites d'étude sont Sorobasso, Kalifabougou, Kodialan et Badogo qui ont été choisis ont été choisis au Sud du Mali en fonction de l'accessibilité, la disponibilité des espèces forestières et le niveau élevé de la malnutrition.

Le site de Sorobasso est situé entre $12^{\circ} 30$ et $12^{\circ} 31$ latitude Nord, entre $5^{\circ} 15$ et $5^{\circ} 16$ longitude Ouest dans le cercle de Koutiala en zone soudanienne. Il reçoit en moyenne $876,60 \mathrm{~mm}$ de pluie par an. La température moyenne annuelle est de $27,2{ }^{\circ} \mathrm{C}$ (Traoré et al. 2003). La végétation ligneuse est composée de Diospyros mespiliformis, Lannea acida, Lannea velutina, Parkia biglobosa, Prosopis africana, Pterocarpus lucens, Saba senegalensis, Sclerocarya birrea et Vitellaria paradoxa.

Le site de Kalifabougou se trouve entre $11^{\circ} 26$ et $11^{\circ} 27$ latitude Nord et $5^{\circ} 57$ longitude Ouest dans le cercle de Sikasso en zone soudanienne. La pluviométrie moyenne annuelle est de 11092,2 mm, avec une température moyenne annuelle de $27,2{ }^{\circ} \mathrm{C}$ (Traoré et al. 2003). La végétation ligneuse est composée de Borassus aethiopum, Cassia sieberiana, Daniellia oliveri, Detarium microcarpum, Pericopsis laxiflora, Pteleopsis suberosa, Piliostigma thonningii, Terminalia macroptera et Vitellaria paradoxa.

Le site de Kodialan est situé entre $11^{\circ} 38$ et $11^{\circ} 40$ latitude Nord, $6^{\circ} 56$ longitude Ouest dans le cercle de Bougouni en zone guinéenne. Il reçoit en moyenne $1196,98 \mathrm{~mm}$ avec une température moyenne annuelle de $27,5{ }^{\circ} \mathrm{C}$ 
(Traoré et al. 2003). La végétation ligneuse est composée de Combretum glutinosum, Detraium microcarpum, Isoberlinia doka, Lannea acida, Terminalia laxiflora et Vitellaria paradoxa.

Le site de Badogo se trouve entre $11^{\circ}$ et $11^{\circ} 01$ latitude Nord, entre $8^{\circ} 11$ et $8^{\circ} 13$ longitude Ouest dans le cercle de Yanfolila en zone guinéenne. La pluviométrie moyenne annuelle est de $1200 \mathrm{~mm}$ avec une température moyenne annuelle de $28^{\circ} \mathrm{C}$. La végétation est composée de Daniellia oliveri, Detarium microcarpum, Entada africana, Isoberlinia doka, Lannea acida, Lannea microcarpa, Lophira lanceolata, Parinari curatellifolia et Pterocarpus erinaceus.

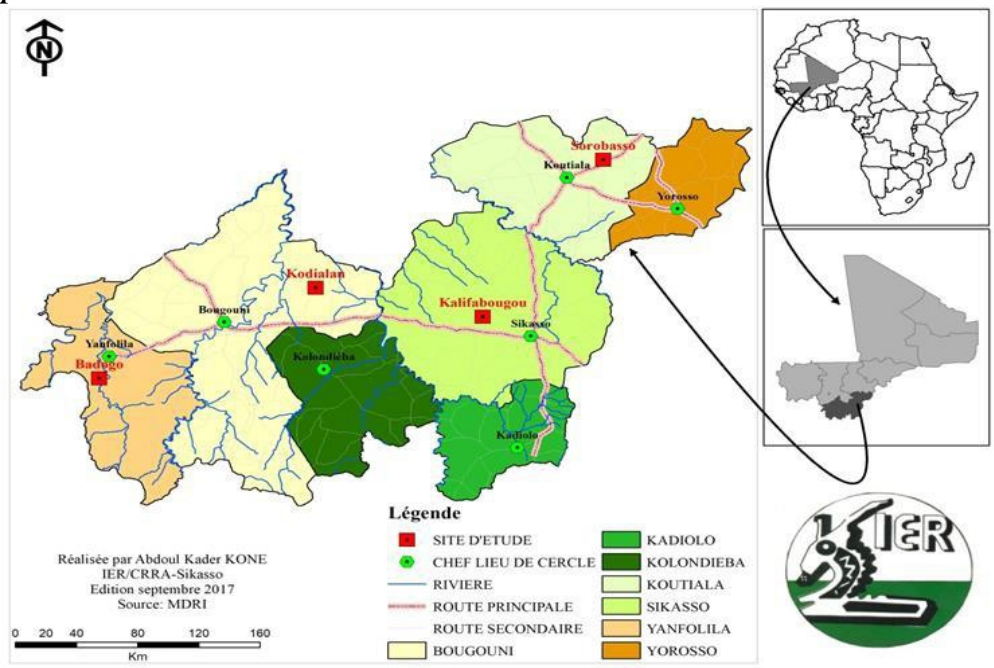

Figure 1 : Localisation des sites d'étude

\section{Inventaire floristique}

L'inventaire des espèces forestières locales alimentaires a été réalisé dans les massifs forestiers naturels suivant l'échantillonnage systématique à un degré, soit deux massifs par zone agro-écologique. Ce type d'inventaire consiste à délimiter des unités primaires ou placettes à l'intérieur desquelles les mesures sont effectuées. Dans chaque massif, la collecte des données a été faite à l'intérieur de 10 placettes de $50 \mathrm{~m}$ x $50 \mathrm{~m}$ avec une équidistante de 50 m suivant la méthode décrite par plusieurs auteurs (Kelly, 2006 ; Glèlè Kakaï et al., 2009). Les mesures ont été portées sur des individus de diamètre supérieur à $3 \mathrm{~cm}$ de toutes espèces confondues et particulièrement celles utilisées dans l'alimentation humaine. Les paramètres mesurés sont le diamètre à hauteur de poitrine (d.b.h) à l'aide de compas forestier et la hauteur totale à l'aide de règle coulissante. Pour chaque espèce rencontrée, le nom de l'espèce et sa famille ont été déterminés. La nomenclature adoptée est celle de l'APG IV (2016). Pour permettre le calcul du taux de régénération, des tiges de diamètre inférieur à $3 \mathrm{~cm}$ ont été dénombrées dans les placeaux de $5 \mathrm{~m}$ sur 
$5 \mathrm{~m}$ qui ont été disposés au centre et aux angles de chacune des placettes décrites ci-dessus, suivant un échantillonnage systématique à deux degrés (Figure 2), soit un total de 40 placettes dans l'ensemble des massifs forestiers.

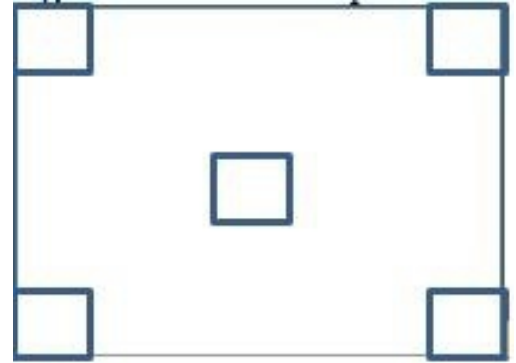

Figure 2 : Disposition des placeaux de collecte de la régénération naturelle

\section{Analyse des données}

Les données collectées ont permis de déterminer la fréquence absolue par simple comptage, l'abondance, la densité à l'hectare, la surface terrière et le taux de régénération. Les variables abondance, densité à l'hectare, diamètre à hauteur de poitrine, hauteur totale et surface terrière ont fait l'objet d'Analyse Normale de Variance (ANOVA).

L'abondance est le rapport du nombre total d'individus d'une espèce sur le total des individus de l'ensemble des espèces rencontrées.

\section{Indices de diversité alpha}

La richesse spécifique, la diversité spécifique de Shannon $\left(H^{\prime}\right)$ et l'équitabilité de Piélou $(E)$ ont été calculées pour chaque site. La richesse spécifique, la diversité spécifique de Shannon $\left(H^{\prime}\right)$ et l'équitabilité de Piélou $(E)$ ont été calculés pour chaque site.

L'indice de Shannon est un indice de diversité qui mesure la composition en espèces d'un peuplement en tenant compte de la richesse spécifique et de l'abondance relative des espèces (Felfili et al., 2004). Il est utilisé pour exprimer la diversité d'un site. Il est principalement déterminé par les espèces dominantes (Hakizimana, 2012).

$$
H^{\prime}=-\sum_{i=1}^{s} p_{i} \log _{2} p_{i}
$$

L'indice d'équitabilité de Piélou (1966) est encore appelé indice de régularité ou d'équi-répartition. Il traduit la manière dont les individus sont distribués à travers les espèces (Adjakpa et al., 2013) et permet de dire si un espace est dominé par une quelconque espèce (Huston, 1994; Dajoz, 2003; Frontier et al., 2008). 


$$
E=\frac{H^{\prime}}{\log _{2} S}
$$

$\mathrm{S}=$ nombre total d'espèces ;

$\mathrm{pi}=(\mathrm{nj} / \mathrm{N})$, fréquence relative des espèces ;

$\mathrm{nj}$ = fréquence relative de l'espèce $\mathrm{j}$ dans l'unité d'échantillonnage ;

$\mathrm{N}=$ somme des fréquences relatives spécifiques ;

Plus l'indice est élevé, plus la diversité est grande.

\section{Données dendrométriques}

La densité est le nombre moyen de tiges sur pied estimé à l'hectare : $D=\frac{N a}{S}$

$\mathrm{D}:$ la densité à l'hectare ;

$\mathrm{N}_{\mathrm{a}}$ : le nombre total d'arbres de l'espèce ;

$\mathrm{S}$ : la superficie couverte (ha).

La surface terrière $G$ est la somme des sections transversales de tous les individus des espèces $\left(\mathrm{m}^{2} / \mathrm{ha}\right)$ :

$$
\mathrm{G}=\frac{\pi}{40000 \mathrm{~s}} \sum_{\mathrm{i}=1}^{\mathrm{n}} \mathrm{d}_{\mathrm{i}}^{2}
$$

di : le diamètre de la tige $\mathrm{i}(\mathrm{cm})$ et $\mathrm{s}$ la superficie couverte (ha).

Le taux de régénération est le rapport entre l'effectif total des jeunes plants (diamètre $<3 \mathrm{~cm}$ ) et l'effectif total du peuplement (Poupon, 1980). L'effectif total du peuplement regroupe les jeunes plants et les plants adultes.

Pour comprendre la structure des espèces forestières locales, la distribution théorique de Weibull a été appliquée sur un échantillon d'espèces présentant un effectif important. Nous avons pris des classes de $10 \mathrm{~cm}$ d'amplitude. Les classes vont de 3-13; 13-23 ; 23-33 ; 33-43, etc. La fonction de densité de probabilité $f(x)$ a été calculée à partir de la formule suivante (Rondeux, 1999) :

$$
f(x)=\frac{c}{b}\left(\frac{x-a}{b}\right)^{c-1} \exp \left[-\left(\frac{x-a}{b}\right)^{c}\right]
$$

$\mathrm{x}$ : diamètre, hauteur ou circonférence à hauteur de poitrine ou hauteur des arbres ; $\mathrm{f}(\mathrm{x})$ : densité de probabilité ; $\mathrm{a}$ : paramètre de position ; $\mathrm{b}:$ paramètre d'échelle ou de taille ; $\mathrm{c}$ : paramètre de forme lié à la structure observée.

\section{Résultats}

\section{Caractéristiques Floristiques}

Les relevés effectués dans les 40 placettes réalisées dans les massifs forestiers ont permis de recenser au total 89 espèces appartenant à 74 genres et 29 familles (tableau 1). L'effectif des familles et des genres varie d'un site d'étude à un autre. Les familles les plus représentées sont les Fabaceae (26 espèces), les Combretaceae (10 espèces), les Rubiaceae (6 espèces) et les 
Anacardiaceae (6 espèces). Celles communes aux quatre sites d'étude sont les Anacardiaceae, les Apocynaceae, les Combretaceae, les Ebenaceae, les Fabaceae, les Meliaceae et les Sapotaceae. Le nombre de genres le plus élevé par famille est enregistré chez les Fabaceae (21 genres).

Parmi les 89 espèces recensées, 66 espèces $(74,16 \%)$ se trouvent à Kalifabougou, 25 espèces $(28,09 \%)$ à Sorobasso, 54 espèces à Kodialan $(60,67 \%)$ et 52 espèces $(58,43 \%)$ à Badogo. L'abondance des espèces varie de $1,42 \%$ à Kalifabougou à $4 \%$ à Sorobasso. Les espèces les plus abondantes sont Vitellaria paradoxa $(48,99 \%$ à Sorobasso), Isoberlinia doka $(15,49 \%$ à Badogo), Pterocarpus lucens (14,09\% à Sorobasso), Detarium microcarpum (12,78 \% à Badogo), Lannea acida (11,93\% à Kodialan), Terminalia laxiflora et Terminalia macroptera (8,42 \% à Kodialan), et Borassus aethiopum $(8,12$ $\%$ à Kalifabougou). Sur les 89 espèces recensées, les espèces alimentaires représentent 34,83\% et se répartissent comme suit : 74,19\% à Kalifabougou ; $32,58 \%$ à Sorobasso ; 67,74\% à Kodialan et 58,06 \% à Badogo.

Les espèces alimentaires communes aux quatre sites d'étude sont Diospyros mespiliformis, Lannea acida, Lannea velutina, Parkia biglobosa, Saba senegalensis et Vitellaria paradoxa. Par contre, les espèces alimentaires présentes dans un seul site sont Adansonia digitata, Borassus aethiopum, Cordia myxa, Lophira lanceolata, Sarcocephalus latifolius et Spondias monbin. L'analyse de variance montre que l'abondance n'est pas significativement différente $(p=0,103)$ entre les massifs forestiers des quatre sites (tableau 1). 
Tableau 1 : Abondance des taxons recensés dans les massifs forestiers au Sud du Mali

\section{Familles $\quad$ Espèces}

Anacardiaceae

Lannea acida*

Lannea microcarpa*

Lannea velutina*

Ozoroa insignis

Sclerocarya birrea*

Spondias monbin*

Annonaceae Annona senegalensis*

Hexalobus

monopetalus*

Apocynaceae Saba senegalensis*

Holarrhena floribunda

Landolphia heudelotii*

Arecaceae

Balanitaceae

Bignoniaceae

Capparaceae

Celastraceae

Chrysobalanac

eae

Combretaceae
Borassus aethiopum*

Balanites aegyptiaca*

Stereospermum

kunthianum

Boscia salicifolia

Boscia senegalensis

Maytenus senegalensis

Parinari curatellifolia*

Anogeissus leiocarpa

Combretum glutinosum

Combretum

micranthum

Combretum molle

Combretum nigricans

Guiera senegalensis

\begin{tabular}{|c|c|c|c|}
\hline \multicolumn{4}{|c|}{ Abondance (\%) } \\
\hline Kalifabougou & Sorobasso & Kodialan & Badogo \\
\hline 1,92 & 3,36 & 11,93 & 5,63 \\
\hline 1,69 & - & 0,82 & 3,36 \\
\hline 1,69 & 0,67 & 1,87 & 1,84 \\
\hline 0,23 & - & 0,35 & - \\
\hline- & 1,34 & 0,12 & - \\
\hline 0,11 & - & - & - \\
\hline 0,90 & - & 0,70 & - \\
\hline- & - & 1,05 & 0,76 \\
\hline 0,11 & 6,04 & 0,94 & 1,30 \\
\hline- & - & 0,58 & - \\
\hline 0,11 & - & - & 0,11 \\
\hline 8,12 & - & 0,47 & - \\
\hline- & - & 0,12 & - \\
\hline- & - & 0,12 & 0,22 \\
\hline 0,11 & - & - & - \\
\hline- & 0,67 & - & - \\
\hline 0,23 & - & 0,23 & - \\
\hline 1,69 & - & 0,23 & 4,44 \\
\hline 0,11 & 2,01 & - & - \\
\hline 1,58 & 0,67 & 3,86 & 1,08 \\
\hline 0,11 & - & - & - \\
\hline 0,90 & 0,67 & 2,57 & - \\
\hline 0,56 & 0,67 & 1,29 & 0,54 \\
\hline 0,34 & - & 0,70 & 0,76 \\
\hline
\end{tabular}




\section{Familles}

Espèces

Combretaceae

Terminalia

avicennioides

Terminalia laxiflora

Terminalia macroptera

Ptelopsis suberosa

Dipterocarpaceae Monotes kerstingii

Ebenaceae

$$
\text { Diospyros }
$$

mespiliformis*

Fabaceae

Piliostigma thonningii

Acacia macrostachya

Acacia nilotica

Acacia seyal

Acacia sieberiana

Acacia sp.

Pericopsis laxiflora

Afzelia Africana

Albizia malacophylla

Burkea Africana

Cassia sieberiana

Cordyla pinnata*

Daniellia oliveri

Detarium

microcarpum*

Dichrostachys

glomerata

Entada Africana

Erythrina senegalensis

Isoberlinia doka
Tableau 1 (Suite 1)

\section{Kalifabougou}

0,23

1,13

7,55

4,85

$-$

1,01

12,85

0,23

0,56

0,11

-

4,17

-

0,34

0,11

4,62

-

4,28

5,98

1,47

3,27

3,38
Abondance (\%)

\section{Sorobasso}

Kodialan

8,42

0,65

2,17

0,11

1,19

0,43

2,22

1,99

2,06

2,71

0,67

0,58

0,35

0,23

1,34

- 1,40

0,22

0,22

0,33

0,43

0,67

5,26

0,35

1,17

5,42

6,67

12,78

0,67

0,23

2,69

6,18

0,65

9,01

15,49

Tableau 1 (Suite 2)

\section{Familles Espèces}

Fabaceae

\section{Kalifabougou}

0,11

2,59

1,92

1,13

\section{Abondance (\%)}

Sorobasso

Kodialan

Badogo

2,49

6,50

4,55

6,71

0,94

0,12

1,29

Pterocarpus erinaceus

Pterocarpus lucens

14,09

1,29


Swartzia madagascariensis

Tamarindus indica*

$$
0,67
$$

0,12

Xeroderris chevalieri

0,56

Loganiaceae

Strychnos spinosa*

0,45

0,35

Malvaceae

Grewia bicolor

Sterculia setigera

0,23

Adansonia digitata*

0,11

Cola cordifolia*

0,11

Grewia mollis

0

2,46

Bombax costatum*

0,34

0,67

0,33

Meliaceae

Khaya senegalensis

0,34

Trichilia emetica

0,45

0,35

Ficus capensis

0,12

0,22

Ficus ovota

0,11

Ficus sur

0,11

0,65

Ochnaceae Lophira lanceolata*

0,23

4,44

Opiliaceae

Opilia celtidifolia

0,33

Phyllanthaceae

Hymenocardia acida

Tableau 1 (Suite 3)

\section{Familles Espèces}

\section{Kalifabougou}

Bridelia ferruginea

Uapaca togoensis

Rhamnaceae

Rubiaceae

Boraginaceae

Rutaceae
Ziziphus mauritiana*

Gardenia erubescens*

Keetia cornelia

Crossopteryx

febrifuga

Feretia apodanthera

Sarcocephalus

latifolius*

Pavetta crassipes

Cordia myxa

Zanthoxylum

zanthoxyloides
1,58

Abondance (\%)
Sorobasso

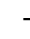

0,12

0,67

0,23 


\begin{tabular}{|c|c|c|c|c|c|}
\hline Salicaceae & $\begin{array}{l}\text { Flacourtia } \\
\text { flavescens* }\end{array}$ & 0,23 & - & 0,23 & - \\
\hline Sapindaceae & $\begin{array}{l}\text { Eriocoelum } \\
\text { kerstingii }\end{array}$ & 0,11 & - & - & - \\
\hline Sapotaceae & Vitellaria paradoxa* & 8,91 & 48,99 & 10,76 & 0,43 \\
\hline \multirow[t]{2}{*}{ Lamiaceae } & Vitex barbata* & 0,23 & - & 0,82 & - \\
\hline & Vitex doniana* & 0,23 & - & - & 0,11 \\
\hline Olacaceae & Ximenia americana* & 0,56 & 0,67 & - & 0,11 \\
\hline Total & & 100 & 100 & 100 & 100 \\
\hline Moyenne & & 1,42 & 4 & 1,82 & 1,92 \\
\hline Probabilité & & & & & \\
\hline
\end{tabular}

* : espèce alimentaire ; - : absence

\section{Indices de diversité}

L'analyse des indices de diversité (Tableau 2) a montré que pour l'ensemble des sites étudiés, la diversité est relativement élevée $\left(H^{\prime}=4,99\right)$, avec une richesse spécifique de 89 espèces et une équitabilité de 0,77 . Les massifs forestiers le moins diversifié et le plus diversifié sont observés en zone soudanienne. Le massif forestier le plus diversifié se rencontre à Kalifabougou avec un indice de diversité de Shannon $H^{\prime}=4,8$ bits et une richesse spécifique de 65 espèces. Le site le moins diversifié est celui de Sorobasso avec un indice de diversité $H^{\prime}=2,89$ et une richesse spécifique de 25 espèces.

Tableau 2 : Indice de diversité des espèces rencontrées : H': Indice de diversité de Shannon ; $\mathrm{S}$ : richesse spécifique ; Hmax : Indice de diversité maximale ; E : équitabilité de Piélou

\begin{tabular}{|c|c|c|c|c|c|}
\hline \multirow{2}{*}{ Indices de diversité } & \multicolumn{2}{|c|}{ Zone soudanienne } & \multicolumn{2}{c|}{ Zone guinéenne } & \\
\cline { 2 - 6 } & Kalifabougou & Sorobasso & Kodialan & Badogo & Global \\
\hline $\mathrm{H}^{\prime}$ & 4,80 & 2,89 & 4,61 & 4,54 & 4,99 \\
\hline $\mathrm{S}$ & 65 & 25 & 54 & 52 & 89 \\
\hline $\mathrm{Hmax}$ & 6,02 & 4,64 & 5,75 & 5,70 & 6,48 \\
\hline $\mathrm{E}$ & 0,80 & 0,62 & 0,80 & 0,80 & 0,77 \\
\hline
\end{tabular}

\section{Caractéristiques dendrométriques des espèces forestières locales alimentaires}

Le tableau 3 ci-dessous donne les caractéristiques dendrométriques des espèces forestières locales alimentaires de la zone d'étude. L'analyse de variance a montré que les moyennes de diamètre à hauteur de poitrine ne sont pas significativement différentes $(\mathrm{p}=0,584)$ entre les massifs forestiers. Des différences significatives ont été observées entre les sites pour la densité moyenne à l'hectare $(p=0,048)$, la hauteur moyenne $(p=0,001)$ et la surface terrière moyenne $(\mathrm{p}=0,001)$. Les sites de la zone guinéenne se distinguent par des densités significativement plus élevées. La densité la plus élevée est 
observée au niveau du site de Bogodo (71 arbres/ha) suivi de Kodialan (63 arbres/ha). La plus faible densité est observée au niveau de Sorobasso situé en zone soudanienne avec une densité moyenne de 24 arbres à l'hectare. Les arbres les plus hauts de taille ont été rencontrés à Kodialan et Badogo en zone guinéenne, alors que ceux de petite taille sont observés à Kalifabougou et Sorobasso en zone soudanienne. Contrairement à la hauteur moyenne, la surface terrière la plus élevée est observée à Kalifabougou et à Sorobasso en zone soudanienne (Tableau 3).

Tableau 3 : Caractéristiques dendrométriques des espèces forestières locales alimentaires des sites étudiés

\begin{tabular}{|c|c|c|c|c|c|}
\hline $\begin{array}{l}\text { Zones agro- } \\
\text { écologiques }\end{array}$ & $\begin{array}{c}\text { Massifs } \\
\text { forestiers }\end{array}$ & $\begin{array}{c}\text { Densité } \\
\text { moyenne } \\
\text { (arbre/ha) }\end{array}$ & $\begin{array}{l}\text { Diamètre } \\
\text { moyen à } \\
\text { hauteur de } \\
\text { poitrine } \\
\text { (cm) }\end{array}$ & $\begin{array}{l}\text { Hauteur } \\
\text { moyenne } \\
(\mathbf{m})\end{array}$ & $\begin{array}{c}\text { Surface terrière } \\
\text { moyenne } \\
\left(\mathrm{cm}^{2} / \mathrm{ha}\right)\end{array}$ \\
\hline \multirow{2}{*}{ Soudanienne } & Kalifabougou & $55^{\mathrm{a}}$ & 11,52 & $5,24^{\mathrm{b}}$ & $202,25^{\mathrm{a}}$ \\
\hline & Sorobasso & $24^{\mathrm{b}}$ & 11,73 & $5,16^{\mathrm{b}}$ & $190,64^{\mathrm{a}}$ \\
\hline \multirow{2}{*}{ Guinéenne } & Kodialan & $63^{\mathrm{a}}$ & 10,99 & $5,53^{\mathrm{a}}$ & $143,59^{b}$ \\
\hline & Badogo & $71^{\mathrm{a}}$ & 11,44 & $5,77^{\mathrm{a}}$ & $151,00^{\mathrm{b}}$ \\
\hline \multicolumn{2}{|c|}{ Global } & 53 & 11,42 & 5,42 & 151 \\
\hline \multicolumn{2}{|c|}{ Probabilité } & 0,048 & 0,584 & $0,001 *$ & $0,001 *$ \\
\hline
\end{tabular}

Par colonne, les valeurs portant la même lettre ne sont pas significativement différentes; celles portant des lettres différentes présentent des différences significatives, soit au seuil de $1 \%$, soit à $5 \%$.

\section{Taux de régénération naturelle des espèces forestières locales alimentaires}

La flore de la régénération naturelle dans les massifs forestiers de la zone d'étude est composée de 26 espèces appartenant à 16 familles (Tableau $3)$ dont les plus représentées sont les Anacardiaceae (4 espèces) et les Fabaceae (3 espèces). Les familles représentées par une seule espèce sont les plus nombreuses: Arecaceae, Salicaceae, Ochnaceae, Chrysobalanaceae, Ebenaceae, Loganiaceae, Rutaceae, Salicaceae, Sapotaceae. Le nombre d'espèces en régénération naturelle a varié en fonction des sites : 18 espèces à Kalifabougou, 6 espèces à Sorobasso, 14 espèces à Kodialan, 13 espèces à Badogo. Le taux de régénération des espèces varie d'un site à un autre et diminue au fur et à mesure que l'on se dirige vers les zones humides.

Les forts taux de régénération ont été enregistrés chez Ximenia americana $(61,06 \%)$ à Sorobasso et Vitellaria paradoxa $(18,22 \%)$ à Kalifabougou (Tableau 4). 
Tableau 4: Taux de régénération des espèces forestières locales alimentaires de la zone

Espèces

Annona senegalensis

Hexalobus monopetalus

Bombax costatum

Sterculia setigera

Borassus aethiopum

Detarium microcarpum

Parkia biglobosa

Tamarindus indica

Diospyros mespiliformis

Flacourtia indica

Gardenia erubescens

Sarcocephalus latifolius

Landolphia heudelotii

Saba senegalensis

Lannea acida

Lannea microcarpa

Lannea velutina

Sclerocarya birrea

Lophira lanceolata

Parinari curatellifolia

Strychnos spinosa

Vitellaria paradoxa

Vitex barbata

Vitex doniana

Ximenia americana

Zanthoxylum zanthoxyloüdes

Moyenne
Familles d'étude

Annonaceae

Kalifabougou Sorobasso

Arecaceae

Fabaceae

Ebenaceae

Salicaceae

Rubiaceae

Apocynaceae

Anacardiaceae

Ochnaceae

Chrysobalanaceae

Loganiaceae

Sapotaceae

Lamiaceae

Olacaceae

Rutaceae
3,30

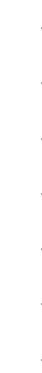

0,24

5,62

3,55

,

4,65

0,49

0,61

0,12

0,37

0,24

2,81

0,24

0,73

0,24

0,12

Taux de régénération (\%)

Kodialan Badogo

$4,90 \quad 7,04$

$0,30 \quad 0,12$

0,91

$10,41 \quad 5,58$

0,48

0,15

0,76

1,46

4,44

0,24

$-$

2,29 0,15

1,52

3,06

0,15

1,53

2,91

- $\quad 0,76$

1,34

-

18,22

7,63

1,07

0,48

3,40

0,24

0,85

-

61,06

1,69

- : absence

\section{Structure en diamètre}

L'analyse de la structure en classe de diamètre des populations de Saba senegalensis, Vitellaria paradoxa, Lannea acida, Lannea microcarpa, Lophira lanceolata, Parkia biglobosa et Borassus aethiopum a montré des tendances variables. L'analyse de la structure en classes de diamètre de la 
plupart de ces espèces a montré une large représentation des individus jeunes au niveau de tous les peuplements. La structure en diamètre des populations de Saba senegalensis, Vitellaria paradoxa, Lannea acida, Lannea microcarpa, Lophira lanceolata et Parkia biglobosa a montré le paramètre de forme $\mathrm{c}$ de la distribution théorique de Weibull proche de 1. Ce type de structure présente une forme en « $\mathrm{J}$ renversé » (Figures 2 à 7 ). Ces espèces présentent une distribution plus ou moins stable.

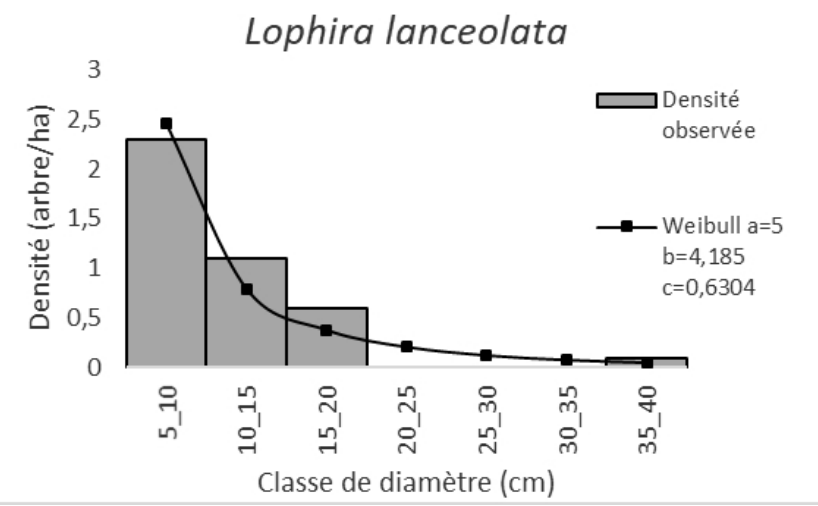

Figure 2 : Structure en classe de diamètre des populations de Lophira lanceolata

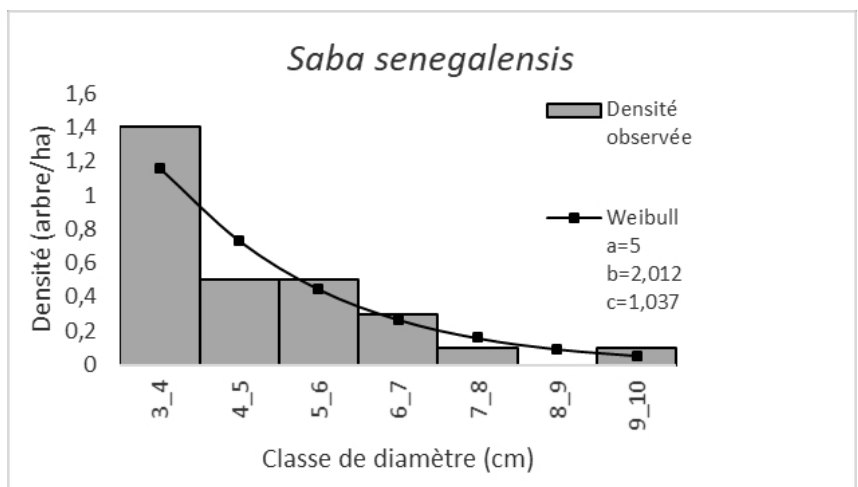

Figure 3 : Structure en classe de diamètre des populations de Saba senegalensis 


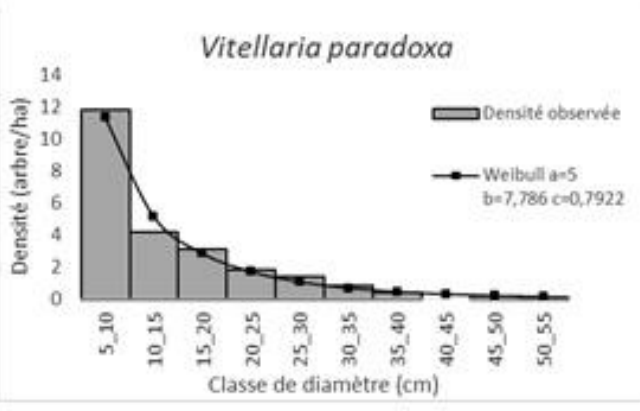

Figure 4 : Structure en classe de diamètre des populations de Vitellaria paradoxa

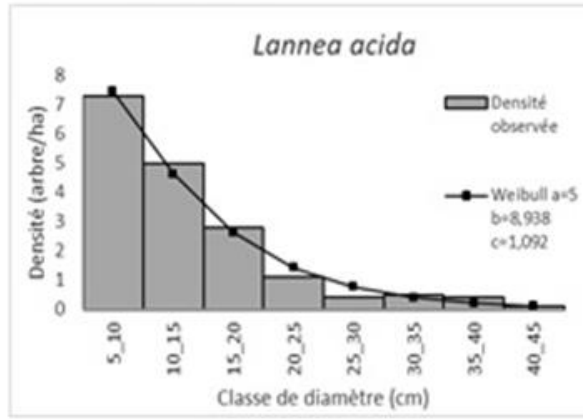

Figure 7 : Structure en classe de diamètre des populations de Lannea acida

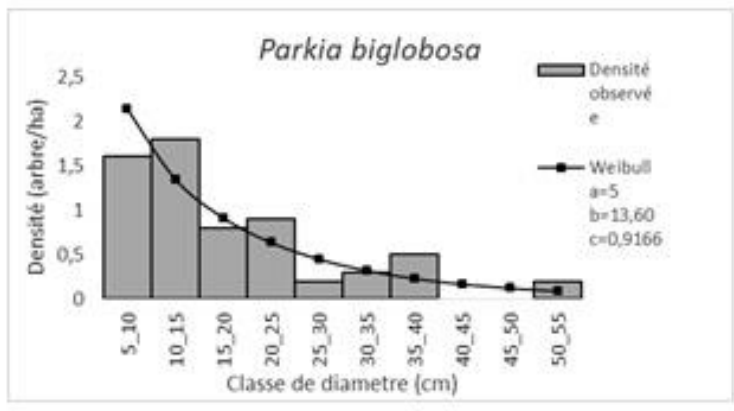

Figure 5 : Structure en classe de diamètre des populations de Parkia biglobosa

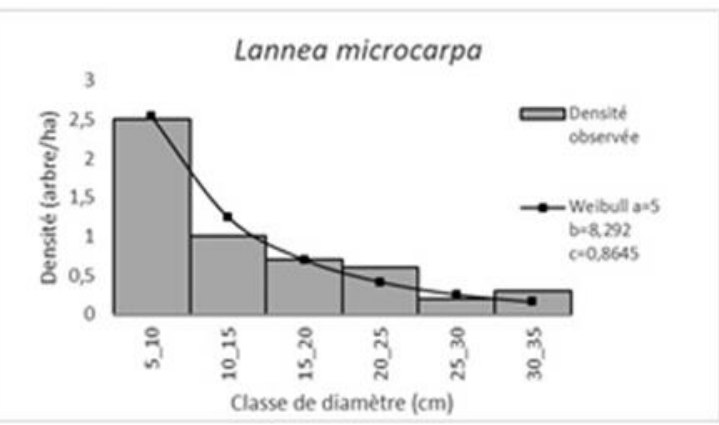

Figure 8 : Structure en classe de diamètre des populations de Lannea microcarpa

Pour la plupart de ces espèces, les classes de diamètre 5 à $10 \mathrm{~cm}$ sont relativement les plus représentées, avec une faible présence d'individus adultes de diamètre supérieur à $15 \mathrm{~cm}$.

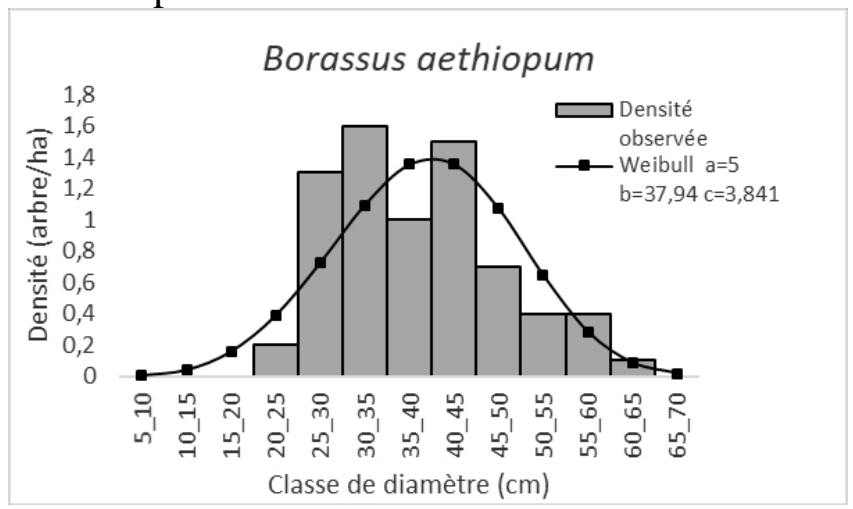

Figure 8 : Structure en classe de diamètre des populations de Borassus aethiopum

Au niveau des populations de B. aethiopum (Figure 8), les individus jeunes et les individus adultes sont faiblement représentés. Les individus de classe de diamètre compris entre 5 et $20 \mathrm{~cm}$ sont totalement absents. Dans ce 
cas, la structure suit une distribution en cloche avec le paramètres de forme c de la distribution théorique de Weibull supérieur à 3,6.

\section{Discussion}

\section{Composition floristique et diversité spécifique}

Les 89 espèces inventoriées dans la zone d'étude représentent 31,45\% des espèces de la région. La prédominance de la famille Fabaceae suivie des Combretaceae dans les massifs forestiers de la région de Sikasso a été confirmée par des études similaires qui ont été réalisées dans la même région (Fadoul, 2013 ; Fagui, 2015). La variation de la richesse spécifique et de l'abondance des espèces forestières dans les massifs forestiers n'a pas suivi le gradient climatique. Les valeurs maximales et minimales ont été enregistrées dans la zone soudanienne. Birnbaum (2017) a abouti à la même conclusion en disant qu'une espèce peu fréquente à l'échelle de la planète peut être extrêmement abondante localement. La faible diversité floristique observée à Sorobasso serait liée à la forte anthropisation (Cuny et Sorg, 2003). La forte abondance de $V$. paradoxa peut être considérée comme une réponse de la société rurale qui la considère comme une espèce prioritaire (Breman et Kessler, 1995 ; Maïga, 2001 ; Sigaud et Eyog-Matig, 2001). La variation de la richesse spécifique constatée s'expliquerait par la variation du climat (Sarr, 2008 ; Yossi et al., 2008), la compétition interspécifique, la disponibilité en ressources et le niveau de perturbations (Palmer, 1994).

\section{Caractéristiques dendrométriques}

L'étude a montré que la densité moyenne des espèces dans les massifs forestiers et dans les champs augmente au fur et à mesure que l'on s'éloigne des zones sèches. Pour tous les massifs forestiers ayant fait l'objet de prospection, la densité enregistrée en zone guinéenne équivaut à environ 1,7 fois celle de la zone soudanienne (Tableau 3). La différence de densité entre les deux zones est due au fait que la zone guinéenne est moins dégradée que la zone soudanienne, ce qui signifie que la densité décroît avec la pression anthropique. Ces résultats sont confirmés par Birnbaum (2017) qui a indiqué qu'actuellement au Mali, les formations arborées de la savane sont soumises aux pressions anthropiques, alors que les formations forestières reliques sont dépendantes d'une forte disponibilité hydrique locale. La forte pression humaine dans les massifs forestiers en zone soudanienne pourrait expliquer la faible présence d'individus reproducteurs à cause des exploitations abusives. Comita et al. (2007) abondent dans le même sens en disant que plus le nombre d'individus reproducteurs est élevé, plus la densité globale est élevée. La valeur élevée de la hauteur moyenne dans les massifs forestiers pourrait s'expliquer par la densité élevée qui occasionnerait une forte concurrence pour la lumière. La valeur élevée des paramètres dendrométriques en zone 
guinéenne pourrait être attribuable aux conditions climatiques qui sont plus favorables.

\section{Régénération naturelle}

La diminution du taux de régénération des espèces dans les massifs forestiers au fur et à mesure que l'on se dirige vers les zones humides serait attribuable à la densité des espèces qui est forte à cet endroit influençant négativement la survie de la régénération naturelle par insuffisance de lumière. Plusieurs travaux de recherche ont indiqué que le fort taux de régénération est enregistré chez les espèces aptes au drageonnage et au marcottage (Douh et al., 2014). Ceci expliquerait la forte régénération de Vitellaria paradoxa (Kelly, 1995 ; Bellefontaine, 2005), de Detarium microcarpum (Kelly, 1995 ; Bationo et al., 2001 ; Bastide et Ouédraogo, 2008) et de Ximenia americana (Fawa et al., 2017).

\section{Structure en diamètre des populations}

La distribution par classes de diamètre a montré des variations en fonction des espèces. Plusieurs travaux réalisés dans les zones sahéliennes et soudaniennes ont confirmé cette variabilité structurale des espèces (Hounkpèvi et al., 2011, Mugasha et al., 2013, Rabiou et al., 2015, Idrissa et al., 2017). Selon la littérature (Herrero-Jáuregui et al., 2012 ; Sandjong Sani et al., 2018), les adaptations aux conditions écologiques, la concurrence pour les ressources et l'exploitation seraient à la base de cette variabilité structurale. La faible présence d'individus adultes de diamètre supérieur à $15 \mathrm{~cm}$ a été observée chez les espèces présentant une forme en $\mathrm{J}$ renversé qui incarne une régénération sûre. Ceci indique que les individus de grandes tailles font l'objet de forte exploitation comme l'ont souligné Idrissa et al. (2017). Ce type de forme est caractéristique des peuplements multi-spécifiques avec prédominance des individus jeunes d'où un fort potentiel de régénération. Le type de forme en « $\mathrm{J}$ renversé » a été observé chez Tamarindus indica (Fandohan et al., 2011), Dialium guineense (Assongba et al., 2013), Prosopis africana (Houètchégnon et al., 2015) et Lophira lanceolata (Lankoandé et al., 2017). La prédominance de jeunes individus peut s'expliquer par la relation qui existe entre le tempérament des espèces et leur distribution en diamètre. Cependant, la survie de ces jeunes individus pose problème à cause des feux de brousse et du surpâturage. Au contraire, les espèces résistant après le passage des feux de brousse présentent une forte proportion d'individus adultes (Nkongmeneck et al., 2010) dans leur distribution, tel est le cas chez Borassus aethiopum dont la distribution est en cloche. Ce type de distribution est caractéristique des peuplements mono-spécifiques à très faible potentiel de régénération. 


\section{Conclusion}

La disponibilité des espèces forestières locales alimentaires a été mise en évidence dans les sites étudiés. L'inventaire a permis de comprendre que les Fabaceae, les Combretaceae, les Rubiaceae, les Malvaceae et les Anacardiaceae sont les familles botaniques les plus représentées dans les massifs forestiers naturels. La diversité des espèces est indépendante de la zone agro-écologique. Les densités à l'hectare des espèces les plus élevées et les arbres les plus hauts de taille se rencontrent dans les sites de la zone guinéenne. La surface terrière la plus élevée et le plus fort taux de régénération naturelle des espèces est observée en zone soudanienne. Les forts taux de régénération ont été enregistrés chez Ximenia americana et Vitellaria paradoxa. Toutefois, l'analyse de la structure en diamètre des populations de Saba senegalensis, Vitellaria paradoxa, Lannea acida, Lannea microcarpa, Lophira lanceolata et Parkia biglobosa a montré l'existence d'un fort potentiel de régénération par opposition aux populations de Borassus aethiopum. Des stratégies doivent être développées pour assurer la conservation, la survie de la régénération naturelle et permettre des activités de propagation à moindre coût des espèces forestières locales alimentaires en vue d'améliorer leur disponibilité et leur compétitivité.

\section{References:}

1. Adjakpa J.P., Yedomonhan H., Ahoton L.E., Weesie P.D.M.\& Akpo L.E. (2013). Structure et diversité floristique des îlots de forêts riveraines communautaires de la Basse vallée de la Sô au Sud-Est du Bénin. Journal of Applied Biosciences, 65 : 4902-4913.

2. APG IV. (2016). An update of the Angiosperm Phylogeny Group classification for the orders and families of flowering plants: APG IV. Botanical Journal of the Linnean Society, 181: 1-20.

3. Assongba Y.F., Djègo G.J. \& Sinsin B. (2013). Distribution des habitats de Dialium guineense (willd) (Fabaceae: Caesalpinioideae) dans les phytodistricts Est du Sud-Bénin. Bulletin Scientifique de l'Institut National pour l'Environnement et la Conservation de la Nature, $12: 1-16$.

4. ARS. (2011). Schéma régional d'aménagement du territoire de Sikasso 2010-2035. Version finale. Région de Sikasso. Mali. 372 p.

5. Bastide B. \& Ouédraogo S.J. (2008). Rejets de Detarium microcarpum et feux précoces. Bois et Forêts des Tropiques, 292(2) : 27-37.

6. Bationo B.A., Ouedraogo S.J. \& Guinko S. (2001). Stratégies de régénération naturelle de Detarium microcarpum dans la forêt classée de Nazinon-Burkina Faso. Fruits, 56(4) : 271-285. 
7. Bellefontaine R. (2005). Pour de nombreux ligneux, la reproduction sexuée n'est pas la seule voie : analyse de 875 cas. Texte introductif, tableau et bibliographie. Sécheresse, $16: 317-328$.

8. Birnbaum P. (2017). Spatialisation de la diversité et de la structure: un enjeu pour la conservation des forêts tropicales. Biodiversité et Ecologie. Université de Montpellier. France. 79 p.

9. Breman H. \& Kessler J.J. (1995). Woody plants in agro-ecosytems of semi-arid regions, with an emphasis on the Sahelian countries. Berlin. Allemagne. Springer Verlag. 340 p.

10. Comita L.S., Condit R. \& Hubbell S.P. (2007). Developmental changes in habitat associations of tropical trees. Journal of Ecology, (95) : 482-492

11. CPS/SDR. (2015). Annuaire statistique 2015 du Secteur du Développement Rural. Ministère de l'Agriculture. Mali. $131 \mathrm{p}$.

12. Cronquist A. (1988). The Evolution and classification of flowering plants. Allen Press. Lawrence. Kansas. 555 p.

13. Cuny P. \& Sorg J.P. (2003). Forêt et coton au sud du Mali : cas de la commune rurale de Sorobasso. Bois et Forêts des Tropiques, 276(2) : $17-31$.

14. Dajoz R. (2003). Précis d'écologie. Dunod. Paris. 615 p.

15. Diarra N., Togola A., Denou A., Willcox M., Daou C. \& Diallo D. (2016). Étude ethnobotanique des plantes alimentaires utilisées en période de soudure dans les régions Sud du Mali. Int. J. Biol. Chem. Sci. 10(1): 184-197.

16. DNEF (2014). Stratégie nationale et plan d'action pour la diversité biologique. Ministère de l'Environnement, de l'Eau et de l'Assainissement. Mali. $150 \mathrm{p}$.

17. DREF (2013). Rapport annuel. Région de Sikasso. Mali. 80 p.

18. DRPIA (2019). Rapport annuel 2018 de la Direction Régionale des Productions et Industries Animales. Sikasso. Mali. 60 p.

19. Douh C., Daïnou K., Loumeto J.J., Fayolle A. \& Doucet J.L. (2014): Explorer la banque de graines du sol pour mieux comprendre la dynamique de régénération des forêts tropicales africaines (synthèse bibliographique), Biotechnologie, Agronomie, Société et Environnement, 18(4) : 558-565.

20. Dury S. \& Bocoum I. (2012). Le «paradoxe» de Sikasso (Mali) : pourquoi « produire plus » ne suffit-il pas pour bien nourrir les enfants des familles d'agriculteurs ? Cahiers Agricultures, 21(5) : 324-336.

21. Fandohan B., Assogbadjo A.E., Glèlè Kakaï R. \& Sinsin B. (2011). Geographical distribution, tree density and fruit production of Tamarindus indica L. (Fabaceae) across three ecological regions in Benin. Fruits, 66(1) : 53-62. 
22. Fadoul Y.O. (2013). Evaluation du potentiel ligneux des formations naturelles de la forêt classée de Farako au Sud du Mali. Mémoire de fin de cycle ingénieur. IPR/IFRA de Katibougou. Mali. 48 p.

23. Fagui (2015). Répertoire des espèces forestières des régions de Kayes, Koulikoro, Sikasso et Ségou. Mali. 339 p.

24. Felfili J.M., Silva Junior M.C., Sevilha A.C., Fagg C.W., Walter B.M.T., Nogueira P.E. \& Rezende A.V. 2004. Diversity, floristics and structural patterns of cerrado vegetation in central Brazil. Plant Ecology, $175: 37-46$.

25. Glèlè Kakaï R., Assogbadjo A.E., Sinsin B. \& Pelz D. (2009). Structure spatiale et régénération naturelle de Pterocarpus erinaceus Poir en zone soudanienne au Bénin. Revue Ivoirienne des Sciences et Technologie, $13: 199-212$.

26. Fawa G., Mapongmetsem M.P., Noubissie-Tchiagam J.B. \& Bellefontaine R. (2017). "Multiplication végétative de Ximenia americana 1. par drageonnage », VertigO - la revue électronique en sciences de l'environnement [En ligne], Regards / Terrain, mis en ligne le 25 mai 2017, consulté le 18 décembre 2019. URL : http://journals.openedition.org/vertigo/18420.

27. Frontier S., Pichod-Viale D., Leprete A., Davoult D. \& Luczak C. (2008). Ecosystèmes : structure, fonctionnement, évolution. 4è édition. Dunod. Paris. 558 p.

28. Hakizimana P. (2012). Analyse de la composition, de la structure spatiale et des ressources végétales naturelles prélevées dans la forêt dense de Kigwena et dans la forêt claire de Rumonge au Burundi. Thèse de doctorat. Université Libre de Bruxelles.

29. Herrero-Jáuregui C., García-Fernández C., Sist P.L. \& Casado M.A. (2012). Recruitment dynamics of two low-density neotropical multiple-use tree species. Plant Ecology, 212(9) : 1501-1512.

30. Houètchégnon T., Gbèmavo D.S.J. Charlemagne O., Christine A.I.N. \& Sokpon N. (2015). Structural characterization of Prosopis africana populations (Guill., Perrott., and Rich.) Taub in Benin. International Journal of Forestry Research, Volume 2015, Article ID 101373. 9 p.

31. Hounkpevi A., Yévidé A.S.I., Ganglo, J.C., Devineau, J.L., Azontonde A.H., Adjakidje V. \& Agbossou E.K. (2011). Structure et écologie de la forêt à Diospyros mespiliformis Hochst. ex A.DC. et à Dialium guineense Willd. de la réserve de Massi (La Lama), Bénin. Bois et Forêts des Tropiques, 308 (2) : 33-46.

32. Huston M.A. (1994). Biological Diversity. The coexistence of species on changing landscapes. Cambridge University Press. 681 p. 
33. Idrissa S., Habou R., Machi II., Mahamane A. \& Mahamane S. (2017): Biodiversity and Structure of Woody Plants of Sahelian Rangelands of Baban Rafi, Niger, International Journal of Biology, 9(4) : 1-9.

34. INSTAT (2016). Enquête nutritionnelle et anthropométrique et de mortalité rétrospective_SMART. Mali. 71 p.

35. Traoré K. \& Birhanu Z.B. (2019). Soil Erosion Control and Moisture Conservation Using Contour Ridge Tillage in Bougouni and Koutiala, Southern Mali. Journal of Environmental Protection, 10 : 1333-1360.

36. Kelly B.A. (1995). Régime de taillis-sous-futaie dans la forêt classée de Farako (résultats après cinq années de suivi). Centre Régional de Recherche Agronomique de Sikasso. Mali. Note technique n¹9.

37. Kelly B.A. (2006). Impact des pratiques humaines sur la dynamique des populations et la diversité génétique de Vitellaria paradoxa (Karité) dans les systèmes agroforestiers au Sud du Mali. Thèse de doctorat. Université de Bamako. Mali. 243 p.

38. Lankoandé B., Ouédraogo A., Boussim J.I. \& Lykke A.M. (2017). Natural stands diversity and population structure of Lophira lanceolata Tiegh. ex Keay, a local oil tree species in Burkina Faso. West Africa. Agroforestry Systems, 91 : 85-96.

39. Maïga A.S. (2001). Situation des ressources génétiques forestières du Mali. Atelier sous-régional FAO/IPGRI/ICRAF sur la conservation, la gestion, l'utilisation durable et la mise en valeur des ressources génétiques forestières de la zone sahélienne (Ouagadougou, 22-24 sept. 1998). Document FGR/9F. FAO. Rome. Italie. 13 p.

40. Nkongmeneck B.A., Kemeuze V.A., Mapongmetsem P.M., Ibrahima A. \& Jiofack R.T. (2010). Distribution des Combretums en rapport avec l'aridité au Cameroun, International Journal of Environmental Studies, 67(1) : 41-50.

41. Palmer M. (1994). Variation in species richness: toward a unification of hypotheses. Folia Geobot. Phytotax, 29 : 511-530.

42. Pielou E.C. (1966). The measurement of diversity in different types of biological collections. Journal of Theoretical Biology, 13 : 131-144.

43. Poupon H. (1980). Structure et dynamique de la strate ligneuse d'une steppe sahélienne au nord du Sénégal. Orstom éd. (Etudes, et Thèses). Paris. 307 p.

44. Rabiou H., Diouf, A., Bationo B.A., Segla K.N., Adjonou K., Kokuste A.D., Radji R., Kokou K., Mahamane A. \& Saadou M. (2015). Structure des peuplements naturels de Pterocarpus erinaceus Poir. dans le domaine soudanien, au Niger et au Burkina Faso. Bois et Forêts des Tropiques, 325(3) : 71-83.

45. Rondeux J. (1999). La mesure des peuplements forestiers. Presses agronomiques de Gembloux. 522 p. 
46. Sandjong Sani R.C., Ntoupka M., Vroumsia T. \& Ibrahima A. (2018). Caractérisation structurale de la végétation ligneuse du Parc National de Mozogo-Gokoro (Cameroun). Flora et Vegetatio Sudano-Sambesica, (21) : 724.

47. Sarr M.A. (2008). Variabilité climatique en Afrique de l'Ouest. Dynamique des espaces végétaux à partir d'images satellites. Exemple du Bassin versant du Ferlo (Sénégal). Journée climatologique-Nantes, 57-76.

48. Sigaud P. \& Eyog-Matig O. (2001). Situation des ressources génétiques forestières de la zone sahélienne et nord-soudanienne. Plan d'action sous-régional pour leur conservation et utilisation durable. Note thématique sur les ressources génétiques forestières. Document de travail FGR/2F. FAO. $111 \mathrm{p}$.

49. Sorg J.P. (2000). Gestion de l'arbre et de la forêt en Afrique de l'Ouest sèche : la recherche et le développement au service des paysans. Actes de la Société jurassienne d'émulation, 9-25.

50. Traoré F., Bayoko A., Sanogo B., Diarra B. \& Konaté M. (2003). Elaboration des scénarios du changement climatique pour le Mali. Ministère de l'Environnement. Mali. 97 p.

51. Vinceti B., Ickowitz A., Powell B., Kehlenbeck K., Termote C., Cogill B. \& Hunter D. (2013). La contribution des forêts aux régimes alimentaires durables. Document d'information pour la Conférence internationale sur les forêts pour la sécurité alimentaire et la nutrition. Document FGR/9F, FAO, Rome, Italie, 13 p.

52. Yossi H., Sanogo Z.J.L., Diakité C.H., Kergnan A.O., Ouattara S. \& Soumaré S. 2008. Impacts des investissements dans la gestion des ressources naturelles au Mali. Rapport de synthèse. Etude Sahel. 19 p. 